\title{
Effect of Treatment for CHC on Liver Disease Progression and Hepatocellular Carcinoma Development in African Americans
}

\author{
Naveen Reddy, Paul Naylor*, Zaher Hakim, Redwan Asbahi, Karthik Ravindran, Elizabeth May, \\ Murray Ehrinpreis and Milton Mutchnick
}

Department of Internal Medicine/Division of Gastroenterology, Wayne State University School of Medicine, Harper University Hospital, Detroit, MI, USA

\begin{abstract}
Background and Aims: African Americans (AA) historically have a low response rate to hepatitis $C$ therapies, and there is limited information available for this patient population regarding the development and treatment of chronic hepatitis $\mathrm{C}(\mathrm{CHC})$. The aim of this study was to evaluate liver disease progression and hepatocellular carcinoma (HCC) development in AA with CHC. Methods: Between 1995 and 2008, 246 AA patients with $\mathrm{CHC}$ were identified from a database of patients and followed until 2012-2013 (average 8 years) or the development of HCC after 2008. Results: Viral clearance (intent to treat; sustained virus response (SVR)) was achieved in $15 \%$ of patients with interferon based therapies with or without ribavirin. AA patients who achieved an SVR $(n=22)$ did not develop HCC or new onset cirrhosis, whereas the HCC incidence in untreated AA patients was $23 \%$ (51/203). Patients who achieved an SVR also had improved fibrosis, as defined by the AST Platelet Ratio Index (APRI) and Fibrosis-4 (FIB-4) score, relative to nonresponders and untreated patients. Conclusions: The severity of liver disease at the first visit (except for cirrhosis) correlated with the development of HCC, but because of the overlap in values between patients, these measurements were not useful for predicting individual risk. Since cirrhosis at the first visit was not a predictive factor, treatment with newer antiviral therapies is the best option for reducing the incidence of advanced liver disease and its harmful outcomes in the AA population. (C) 2015 The Second Affiliated Hospital of Chongqing Medical University. Published by XIA \& HE Publishing Ltd. All rights reserved.
\end{abstract}

Keywords: Hepatitis C; Cirrhosis; Hepatocellular carcinoma; APRI; Fib-4. Abbreviations: AA, African Americans; AFP, alpha feto-protein; ALT, alanine aminotransferase; APRI, AST Platelet Ratio Index; AST, aspartate aminotransferase; AUROC, Area Under the Receiver Operating Characteristic Curve; $\mathrm{CHC}$, chronic hepatitis $\mathrm{C}$; $\mathrm{CT}$, computed tomography; DAA, direct acting antiviral; EMR, electronic medical records; ETR, end of treatment response; FIB-4, fibrosis-4; HCC, hepatocellular carcinoma; HCV, hepatitis C virus; IFN, interferon; IRB, institutional review board; ITT, intent to treat; MRI, magnetic resonance imaging; RNA, ribonucleic acid; SVR, sustained virus response; UPG, University Physician Group.

Received: 22 April 2015; Revised: 14 May 2015; Accepted: 18 May 2015 DOI: $10.14218 / \mathrm{JCTH} .2015 .00013$.

*Correspondence to: Paul Naylor, Gastroenterology 6 Hudson Bldg, Harper University Hospital, 3990 John R, Detroit, MI 48201, USA. Tel: +1-313-745-8601, Fax: +1-313-745-8843, E-mail: pnaylor@med.wayne.edu

\section{Introduction}

Hepatitis $\mathrm{C}$ virus (HCV) related morbidity and mortality risk is significant in patients with chronic hepatitis $\mathrm{C}(\mathrm{CHC})$, which affects approximately 3.2 million people in the United States ( $1 \%$ of the population). ${ }^{1-5}$ In patients treated with interferon (IFN) and ribavirin, 25-60\% achieved sustained virus response (SVR), and with the addition of first generation direct acting antivirals (DAAs), SVR was achieved in almost $80 \% .^{6-9}$ Treatment with new DAAs led to SVR rates greater than $90 \%{ }^{10-12}$ African Americans (AA) are twice as likely to be infected with HCV relative to the nonHispanic-Caucasian US population and have historically had lower response rates to approved IFN based therapies. ${ }^{4-20}$ Although fibrosis progression is less rapidly in AA compared to Caucasians, it is unlikely to be a good clinical strategy to counsel $A A$ that they are at lower risk for rapidly developing advanced liver disease. ${ }^{19-21}$ Thus, more specific information about the AA population is needed to better understand the progression of liver disease, especially after achieving viral clearance, and to identify optimal treatment strategies.

A number of publications have compared the response and outcomes in patients treated with IFN-based therapies. ${ }^{22-27}$ All studies have demonstrated that patients who achieve an SVR have better outcomes with respect to cirrhosis, development of HCC, and all-cause mortality. A report by Cozen et al compared outcomes for treated and nontreated patients in two representative diverse populations. The results suggested that patients who received IFN-based therapies but failed to clear HCV may be at increased risk for developing cirrhosis compared to untreated patients. Overall survival was not significantly different. ${ }^{28}$ Additional studies provided conflicting results, but these did not contain a significant $A A$ population. Finally, a study utilizing electronic medical records (EMRs) from a Department of Veterans Affairs Clinical Registry confirmed the value of achieving SVR and found that AA were at lower risk than Caucasians for all liver events. ${ }^{29}$

The aim of this prospective study was to evaluate the effect of IFN based therapies on outcomes in AA patients. The predominant population in our urban clinic is $A A$, and we previously described the demographics and $\mathrm{HCV}$ relevant disease parameters in this patient group. ${ }^{30}$ Here, we identified 346 AA first seen in 1995-2008 and followed them for 8 years on average. Our findings further the understanding of liver disease progression in AA and provide information that 
will specifically guide clinical management and therapy decisions in this underserved and under-investigated population.

\section{Methods}

In this prospective study for outcomes, we started with screened a database of $3,800 \mathrm{CHC}$ patients who were seen at the Wayne State University Physician Group (UPG) Gastroenterology Clinic between 1995 and 2008. All patients signed an institutional review board (IRB) approved consent form. Using the EMR and an IRB approved Health Insurance Portability and Accountability Act (HIPAA) exemption from UPG and Detroit Medical Center, we identified patients who were diagnosed with HCC between 2009 and 2013 and were in the original database. We also identified patients were seen again between January 2012 and July 2013 in order to identify patients with a significantly long follow up time. In total, we identified 346 AA patients with hepatitis C genotype 1 that were followed for 4 to 18 years. Available patient information included demographic information, laboratory values, biopsies, imaging, and response to treatment. First visit and "early visit" indicate that the information was relevant to the initial assessment of the patients although some data was order and obtained later as part of the initial assessment. Treatment response data was obtained at the follow up visits. Cirrhosis was diagnosed with a combination of liver biopsy, ultrasound, computed tomography (CT), magnetic resonance imaging (MRI), and/or esophagogastroduodenoscopy evidence of portal hypertension. Since repeat biopsies were rarely performed, two well-studied markers of fibrosis were used to determine changes in fibrosis over time. ${ }^{31-34}$ The aspartate amino transferase (AST) platelet ratio index (APRI) was defined as $((\mathrm{AST}(\mathrm{IU} / \mathrm{mL}) / 40 \mathrm{IU} / \mathrm{mL}) /$ Platelets (count $\left.\left.\times 10^{3} / \mathrm{mL}\right)\right) \times 100 .^{33}$ Fibrosis-4 (FIB-4) was defined as $(($ Age $($ years $)) \times($ AST $(\mathrm{IU} / \mathrm{mL}))) /\left(\left(\right.\right.$ Platelets $\left(\right.$ count $\left.\left.\times 10^{3} / \mathrm{mL}\right)\right)$ $\times($ Square root $\mathrm{ALT}(\mathrm{IU} / \mathrm{mL})) .^{34}$ Both assays are continuous variables and the literature cutoffs to define minimal vs significant fibrosis were 0.7 for APRI and 1.45 for FIB-4. For cirrhosis, the cutoffs were 1.0 for APRI and 3.5 for FIB-4. The response of AA to therapy was based on intent to treat (ITT). The SAS based statistical program JMP ${ }^{\circledR}$ was used for statistical analysis. To compare different groups, student's t test, analysis of variance (ANOVA), or chi-square was used, depending on the variable. Changes between early visit and recent visit were evaluated using pairwise analysis. In order to determine the relationship between multiple factors defined in univariate analysis and outcomes, multivariate analysis (logistic regression) was used. $p$ value less than 0.01 was considered statistically significant.

\section{Results}

In total, 346 AA patients, followed for an average of 8 years, were included in our study. Table 1 presents their characteristics as related to their treatment and response. The study group was $57 \%$ male, and the mean age was 54 years with a range consistent with known infection rates of individuals born between 1945 and 1965. The mean levels of alanine aminotransferase (ALT) and AST were elevated, but albumin and platelet levels were within normal range. The majority of patients had liver biopsy (257 out of $346(74 \%)$ ), and the biopsy mean fibrosis score, as defined by the Metavir, system was 1.88 .

All treated patients received IFN based therapies, and 147 patients $(43 \%)$ received at least one dose of treatment. The majority of treated patients ( $86 \%$ ) were given dual therapy of pegylated-IFN and ribavirin. The SVR rate was $15 \%$ based on ITT and $23 \%$ based on patients who adhered to the protocol. When patients who relapsed following treatment were included, the end of treatment response (ETR) was $24 \%$.

Table 1. Characteristics of AA patients with $\mathrm{CHC}$ and treatment-induced changes in liver function tests

\begin{tabular}{|c|c|c|c|c|c|c|c|c|c|c|c|c|}
\hline \multicolumn{13}{|c|}{ A: Characteristics at first visit stratified by treatment and outcomes } \\
\hline & \multicolumn{3}{|c|}{ Time between visits } & \multicolumn{3}{|c|}{ Fibrosis (Metivar) } & \multicolumn{3}{|c|}{ HCV RNA $\left(\times 10^{6} \mathrm{IU} / \mathrm{mL}\right)$} & \multirow{2}{*}{$\begin{array}{l}\text { Gender } \\
\% \text { Male }\end{array}$} & \multirow{2}{*}{\multicolumn{2}{|c|}{$\begin{array}{l}\text { Cirrhosis } \\
\%\end{array}$}} \\
\hline & Mean & \multicolumn{2}{|c|}{ SEM } & Mean & \multicolumn{2}{|c|}{ SEM } & Mean & \multicolumn{2}{|c|}{ SEM } & & & \\
\hline Not Treated & 7.3 & \multicolumn{2}{|c|}{0.2} & 1.7 & \multicolumn{2}{|c|}{0.1} & 1.4 & \multicolumn{2}{|c|}{0.1} & $60 \%$ & \multicolumn{2}{|c|}{$13 \%$} \\
\hline Discontinued & 8.5 & \multicolumn{2}{|c|}{0.5} & 2.4 & \multicolumn{2}{|c|}{0.2} & 1.8 & \multicolumn{2}{|c|}{0.2} & $64 \%$ & \multicolumn{2}{|c|}{$21 \%$} \\
\hline NonResponder & 11.0 & \multicolumn{2}{|c|}{0.4} & 1.8 & \multicolumn{2}{|c|}{0.2} & 1.5 & \multicolumn{2}{|c|}{0.2} & $48 \%$ & \multicolumn{2}{|c|}{$7 \%$} \\
\hline Relapse & 9.9 & \multicolumn{2}{|c|}{0.9} & 1.8 & \multicolumn{2}{|c|}{0.4} & 1.3 & \multicolumn{2}{|c|}{0.4} & $46 \%$ & \multicolumn{2}{|c|}{$23 \%$} \\
\hline SVR & 7.2 & \multicolumn{2}{|c|}{0.7} & 1.7 & \multicolumn{2}{|c|}{0.3} & 0.7 & \multicolumn{2}{|c|}{0.3} & $61 \%$ & \multicolumn{2}{|c|}{$9 \%$} \\
\hline \multicolumn{13}{|c|}{ B: Change between visits stratified by treatment and outcomes } \\
\hline & \multicolumn{3}{|c|}{$\operatorname{ALT}(U / L)$} & \multicolumn{3}{|c|}{ AST (U/L) } & \multicolumn{3}{|c|}{ Albumin (g/dL) } & \multicolumn{3}{|c|}{ Platelets $(\mathrm{K} / \mathrm{mL})$} \\
\hline & Pre & Post & Sig & Pre & Post & Sig & Pre & Post & Sig & Pre & Post & Sig \\
\hline Not Treated & 69 & 65 & NS & 83 & 66 & $*$ & 4.02 & 3.80 & $* *$ & 220 & 198 & $* *$ \\
\hline Discontinued & 68 & 52 & NS & 68 & 47 & $*$ & 4.22 & 3.78 & $* *$ & 217 & 207 & NS \\
\hline NonResponder & 91 & 73 & NS & 73 & 65 & NS & 4.12 & 3.79 & $* *$ & 217 & 198 & NS \\
\hline Relapse & 74 & 43 & NS & 61 & 38 & $*$ & 3.99 & 3.90 & NS & 191 & 185 & NS \\
\hline SVR & 80 & 28 & $*$ & 78 & 24 & $* *$ & 4.03 & 4.02 & NS & 253 & 245 & NS \\
\hline
\end{tabular}

\footnotetext{
${ }^{\#}$ Significance defined by pair wise analysis; NS, not significant, $p>0.01 ; * p<0.01 ; * * p<0.001$.
} 
A. APRI Before and After Treatment

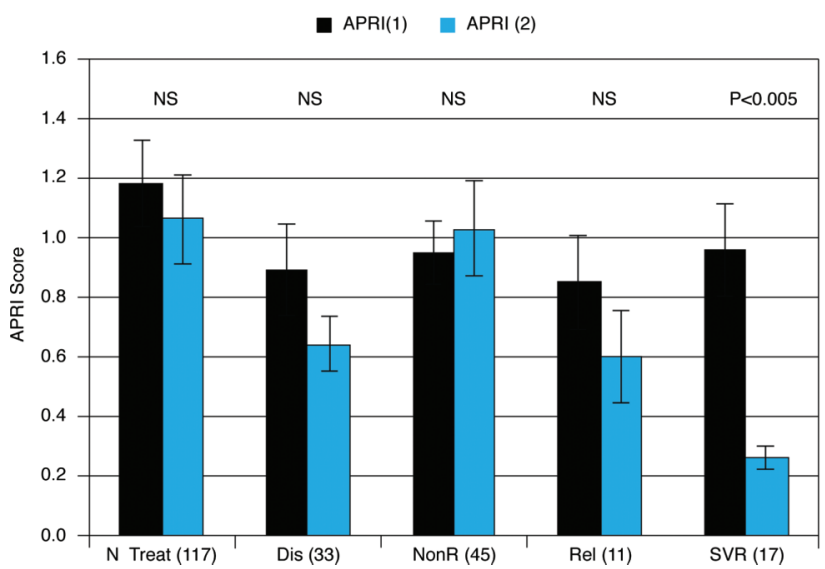

B. FIB-4 Before and After Treatment

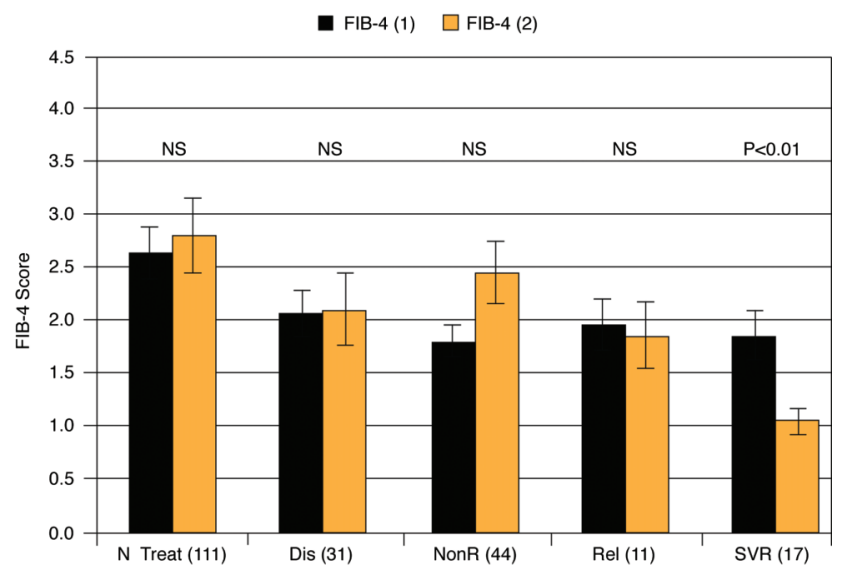

Fig. 1. Fibrosis changes in treated vs. nontreated patients defined by APRI and FIB-4. The fibrosis scores by APRI (A) and FIB-4 (B) are plotted as the average for paired patients between the initial visit (1) and follow-up visit (2). The number of patients in each group is in the parenthesis. $N$ Treat, nontreated; Dis, discontinued; NonR, nonresponders; Rel, relapsed; SVR, sustained virus response. Significance was defined using the paired difference test.

When patients were stratified by treatment, there were minimal differences between various groups of patients at the first visit (Table 1 and Fig. 1). The largest difference, although statistically insignificant, was a reduction in HCV ribonucleic acid (RNA) levels in patients with SVR relative to other groups. In addition, the values before treatment and after treatment/no treatment for liver related laboratory values as a function of response to treatment were compared. The most improved patients were those who achieved an SVR, had reversal of ALT and AST elevation, and had no decline in albumin and platelet counts. APRI and FIB-4 were used to assess changes in fibrosis/cirrhosis. ${ }^{31-34}$ We used biopsy data to validate both the APRI and FIB- 4 in our study population. There was a strong correlation between the degree of fibrosis defined by biopsy and fibrosis as calculated by APRI and FIB-4 $(p<0.001$ data not shown). Also the Receiver Operating Characteristic and the Area Under the Receiver Operating Characteristic Curve (AUROC) values for the AA population were between 0.56 and 0.75 (data not shown). These results are consistent with the literature, and confirm that both calculations are sensitive and specific for measuring differences in fibrosis in AA. ${ }^{31-34}$

When the APRI and FIB-4 were evaluated in patients at the initial visit and follow-up, there was no significant change in fibrosis in patients that were not treated (Fig. 1). In patients who were treated, only the patients who achieved SVR had a significant improvement in these fibrosis scores relative to the other groups. Statistical significance was assessed using a pair-wise analysis, and there were differences in individual patients but not group averages.

Patients who achieved SVR did not develop new cirrhosis or HCC in the follow-up period (Fig. 2). In comparison, patients who were not treated, who discontinued treatment, or who were nonresponsive to treatment developed HCC and newly identified cirrhosis. The nonresponding patients, however, had a lower incidence of HCC than untreated or discontinued treatment patients. Patients achieving an ETR but not SVR did not develop HCC but exhibited a similar incidence of new cirrhosis as the other groups.

Since males are more likely to develop HCC, we compared HCC development and response to therapy by gender (Fig. 3).
The response to treatment was similar for both genders (13\% F vs. $18 \% \mathrm{M})$, although the percentage of females treated $(46 \%)$ was greater than males (38\%). As shown in Fig. 3, AA males were more likely to develop HCC than females, but patients achieving an SVR or ETR with subsequent relapse did not develop HCC regardless of gender. In addition, although males had a higher incidence of HCC, the time to development of HCC was not statistically significant between the genders ( $F=9$ years vs. $M=8$ years).

In order to define the natural history of the disease in AA patients, we determined the time to $\mathrm{HCC}$ in AA patients who were not treated. The time to HCC onset ranged from 1 year to 14 years after the first visit, with 4 to 6 years being the most common timeframe $(n=24$, Fig. 4). There was no

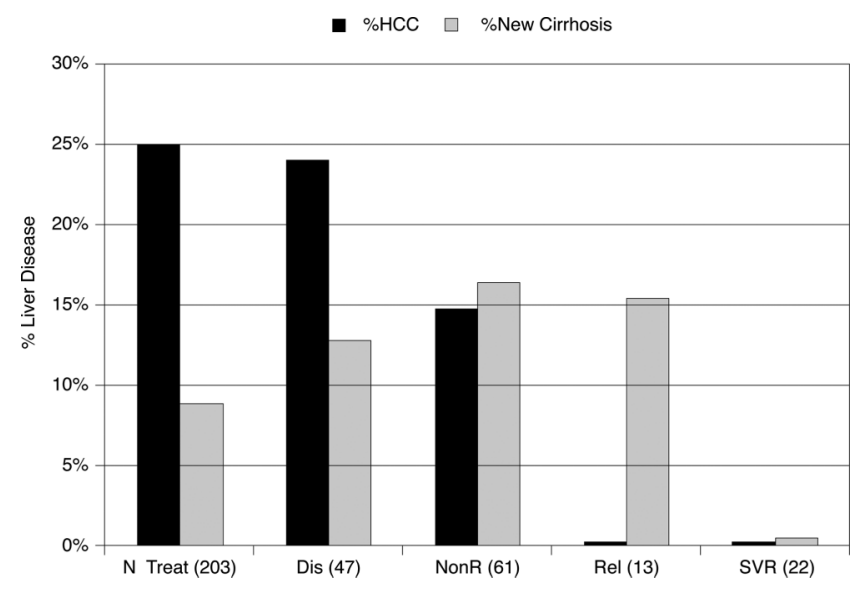

Fig. 2. Advanced liver disease in AA patients with or without treatment at follow-up. Patients were evaluated for the development of hepatocellular carcinoma (HCC) and new cases of cirrhosis at visits following the initial visit. The majority of patients were treated with Peg-IFN + ribavirin $(86 \%$ of treated patients). The SVR was $15 \%$ by ITT and $23 \%$ by protocol. ETR (SVR + relapse patients) was $24 \%$ by ITT and $36 \%$ by protocol. The number of patients in each group is in the parenthesis. N Treat, nontreated; Dis, discontinued; NonR, nonresponders, Rel, relapsed; SVR, sustained virus response. SVR and patients who relapsed after ETR did not develop HCC. 


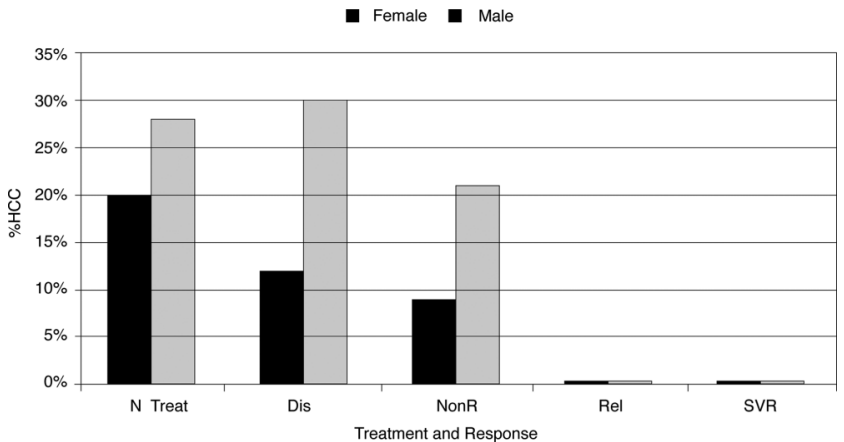

Fig. 3. Patient treatment, response by gender, and development of HCC. The $\%$ of patients who developed HCC was plotted by response to treatment and gender. Similar numbers of AA patients were treated ( $46 \% \mathrm{~F}$ and $38 \% \mathrm{M})$, and response rates based on intent to treat were also similar (15\%). Although males were more likely to develop HCC, HCC did not develop in either the SVR or ETR (relapse) patients, regardless of gender. The average time to HCC was 9 years for females and 8 years for male (nonsignificant by Chi-Square).

difference in time to HCC diagnosis between AA patients with or without cirrhosis at the time of the first visit.

Untreated patients who did not develop HCC were compared to those who did develop HCC in order to determine whether particular parameters at the first visit were useful predictors of HCC development. ALT, AST, albumin, platelet counts, and alpha fetoprotein (AFP) all correlated/inversely correlated with HCC development in AA $(p<0.005$, data not shown). Fig. 5 presents the data related to fibrosis (liver biopsy Metavir scores, APRI, and FIB-4), and all were significant predictors of HCC. Fibrosis but not cirrhosis at the first visit was a significant risk factor for HCC. There was a wide range and considerable overlap in all patient parameters among patients who developed HCC and those who did not. Therefore, the values could not be used to predict individual risk for developing HCC. When using multivariate analysis for all risk factors (ALT, AST, albumin, platelets, AFP, stage of fibrosis, APRI, FIB-4, and cirrhosis), no parameters were identified that were independent predictors of risk for developing HCC when subjected to logistic regression analysis. When the multivariate analysis was restricted to only fibrosis

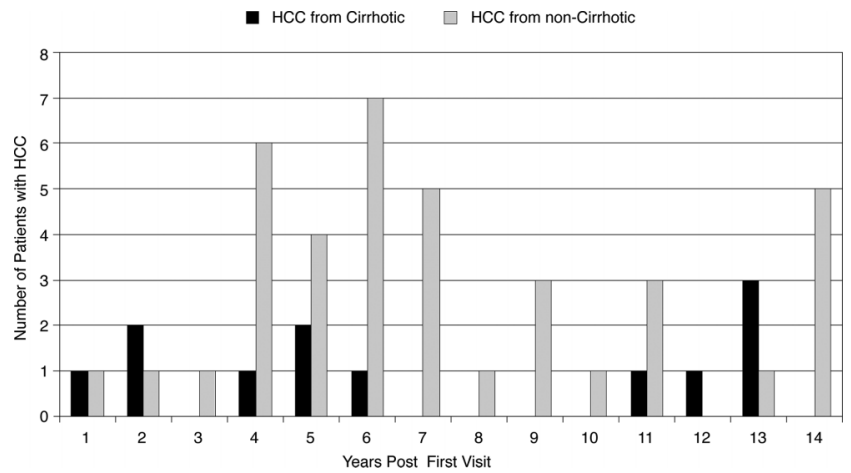

Fig. 4. Number of AA patients diagnosed with HCC plotted by the year of diagnosis. All patients $(n=51)$ who developed HCC were stratified by those who had cirrhosis at first visit (HCC from cirrhotic; $n=12$ ) and those who did not (HCCnoncirrhotic; $n=39$ ). Almost half $(24 / 51=48 \%)$ of patients developed HCC $4-7$ years after the first visit but a significant number of patients $(25 \%)$ did not receive a diagnosis of HCC for more than 11 years. The risk of developing of HCC at the first visit was not predicted by the presence of cirrhosis, since the time to $\mathrm{HCC}$ distribution was similar for those with and without cirrhosis at the first visit. relevant independent predictors (Metavir Stage, APRI, and FIB-4), the only independent predictor of HCC was stage of fibrosis $(p<0.002)$.

\section{Discussion}

In this study, we followed AA patients with $\mathrm{CHC}$ for an average of 8 years who were infected with HCV genotype 1 . CHC outcomes were defined by either the development of cirrhosis, HCC, or fibrosis from the initial visit to the most recent follow up in 2012-2013. As part of this study, we confirmed that a significant number of patients with early visit liver biopsy results had a good correlation with both APRI and FIB-4, thus confirming them as useful surrogate markers for fibrosis in AA patients. Since repeat biopsies were rarely performed, fibrosis was detected using APRI and FIB-4. $7,31-34$ The study demonstrated that permanent viral elimination (defined as SVR) with IFN based treatment in AA patients with $\mathrm{CHC}$ protected them from cirrhosis and $\mathrm{HCC}$ and reversed fibrosis. Although $\mathrm{CHC}$ patients with ETR who relapsed developed new cases of cirrhosis at rates similar to those of nontreated or treatment failure patients, they did not develop HCC. In contrast, patients who failed treatment or were not treated were at high risk for the development of HCC and new onset cirrhosis. This study also confirmed that AA males are at higher risk for developing HCC than AA females. The response to therapy was similar and achieving an SVR reduced the risk of HCC in both genders. Since a significant number of patients developed HCC, we were able to determine whether HCC risk in AA was predictable based on the first clinical visit. Unlike many studies, we plotted individual patients rather than an average in order to assess the clinical value per patient. Regardless of whether serum assays (ALT, AST, AFP, albumin, platelets) or fibrosis assays (biopsy, APRI, FIB-4) were compared between patients who did or did not develop HCC, there was no reliable predictor on an individual basis. This was because of the large variation of values in each group. Somewhat unexpected, we found that cirrhosis at first visit in AA was not predictive of HCC development. When multivariate analysis was used to determine whether there was a single independent factor that was predictive of development of HCC in AA, only fibrosis, as defined by biopsy at the first visit, was even moderately predictive. This lack of positive predictive value for factors was further confirmed by the finding that when patients who developed HCC were stratified by cirrhosis (or no cirrhosis) at first visit, there was no relationship between time to $\mathrm{HCC}$ and cirrhosis.

Although the number of patients achieving an SVR was sufficient for analysis, we could not rule out the possibility of the development of HCC in patients who achieved an SVR. There are a number of publications suggesting that the rate of HCC may be as high as $5 \%$ in patients experiencing an SVR. ${ }^{35-37}$ Those studies are, however, primarily in Asian and European populations and do not contain significant numbers of AA. The primary risk factors for developing $\mathrm{HCC}$ in patients with SVR are males with genotype 1 and cirrhosis at the time of viral clearance. The majority of HCC cases occurred within 3 years, suggesting the presence of small precancerous lesions at the time of viral clearance. Since our population contains patients with these significant risk factors and a high incidence of HCC was present in the untreated and nonresponding population, it is possible that the development of HCC in AA after clearance of the virus is considerably less likely than in the other populations studied. 
A. APRI and Risk of HCC Development

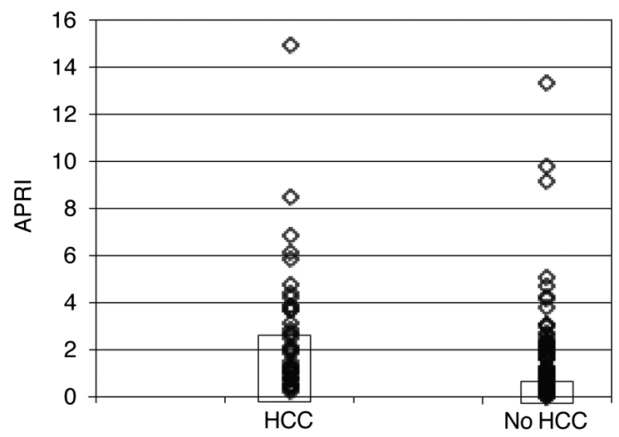

C. Metivar Fibrosis Score and HCC Development

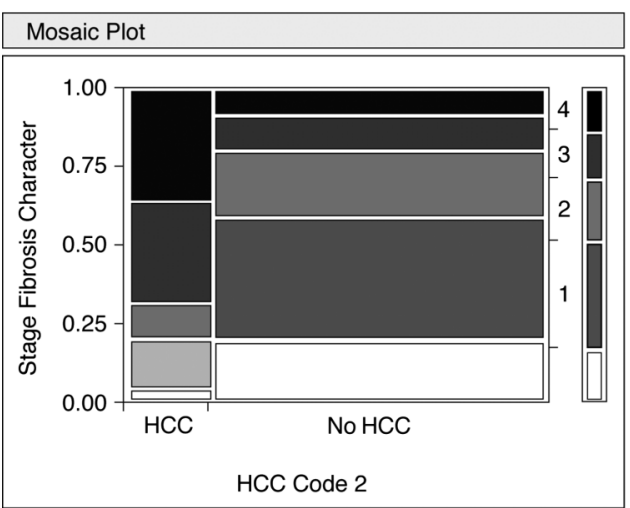

B. FIB-4 and Risk of HCC Development

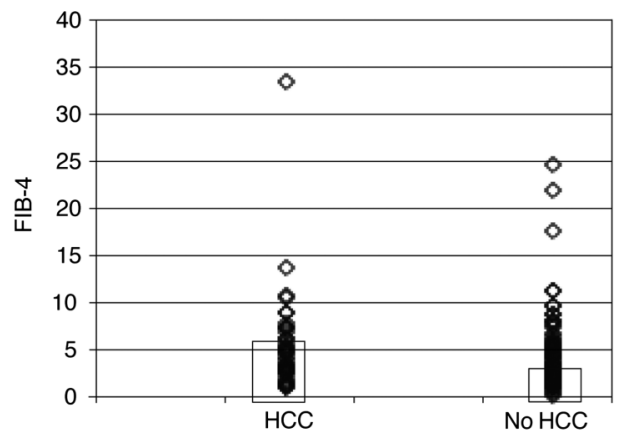

D. Cirrhosis and HCC Development

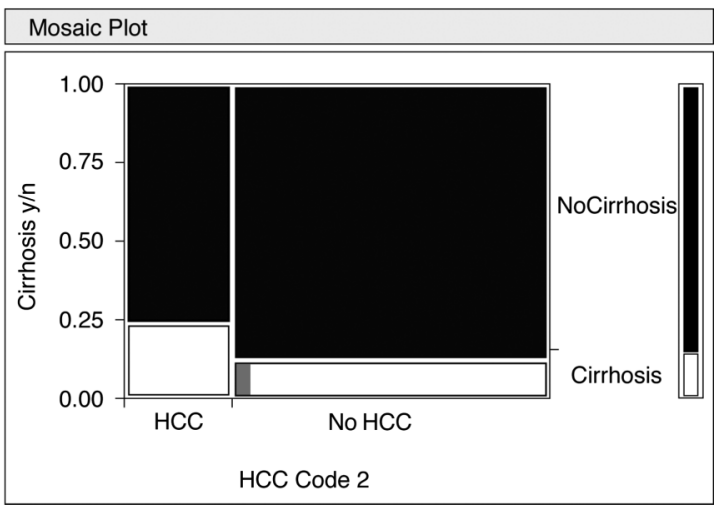

Fig. 5. Fibrosis/cirrhosis relevant predictors of $\mathbf{H C C}$ for individual patients. Individual patients were stratified by those who developed HCC or those who did not and their individual values for potential risk factors at first visit are plotted. For continuous variables, the graphs have the mean and SEM and significance was defined with a student's $t$ test $(A, B)$. For discontinuous values (fibrosis by Metavir and presence/absence of cirrhoses) the data are presented as mosaic plots and significance was calculated using Pierson Chi-square (C, D). The width of the plots reflect the total numbers of patients, and the various shadings represent the \% of patients with each parameter (Scores of $0-4$ or cirrhosis or no cirrhosis). The bar to the right of each graph reflects the \% of total patients. Similar results were obtained for AST, albumin, platelet counts, and AFP; and the difference at entry between HCC and no HCC was significant ( $p<0.001$ for all, data not shown). The wide variability and overlap between individuals in those assays was also similar to that shown in the Figure. Although patients who developed HCC were more likely to have cirrhosis at first visit, the difference was not statistically different.

To a large extent, this study is prospective in nature since patients were first seen prior to 2008 and subsequently seen until 2013 or the development of HCC. Patients who developed HCC were followed for 1-14 years, while those who did not, were seen for a minimum of 4 years and a maximum of 18 years after initial presentation. This study did not compare Caucasians to $A A$, as the number of Caucasians seen in our clinic has continued to decline and there were insufficient numbers available for statistical analyses. The incidence of HCC in AA in our study was $20 \%$ in untreated patients, which was sufficient to provide statistical and clinical relevance to our study.

\section{Conclusions}

Perhaps the most important observation of the study was that AA who did achieve SVR were similar to other groups of SVR patients who also had improved outcomes relative to untreated or nonresponding patients. With the advent of more effective DAA therapies, possible differences in response rates between $A A$ and Caucasians are no longer an issue, and more patients should achieve an SVR. ${ }^{12}$ Although there are many risk factors for the development of HCC in AA patients, if patients are not successfully treated, it remains difficult to predict the development of HCC. Since identification of cirrhosis at an early visit may not be a significant risk factor in AA, surveillance in patients with significant fibrosis, rather than just cirrhosis, is warranted. Unfortunately in many studies to date, it is often a challenge to provide effective HCC surveillance of this population; and thus, elimination of the virus remains the optimal goal of therapy.

\section{Acknowledgements}

Partially supported by an investigator initiated grant (IN-US334-1301) from Gilead Sciences Inc to MM and PN.

\section{Conflict of interest}

None

\section{Author contributions}

Collection of data, analysis of data, interpretation of findings, drafting article (NR); conception of study, data collection and analysis, interpretation, revising of article for important intellectual content, acquisition of funding (PN); collection of 
data $(\mathrm{ZH})$; collection of the data $(\mathrm{RA})$; collection and analysis of the data (KR); data collection, interpreting data and revising of article for important intellectual content (EM); revising of the article for important intellectual content (ME); assisting with the design and conception of study, interpretation and revising of article for important intellectual content, acquisition of funding (MM).

\section{References}

[1] Averhoff FM, Glass N, Holtzman D. Global burden of hepatitis C: considerations for healthcare providers in the United States. Clin Infect Dis 2012;55: S10-S15. doi: 10.1093/cid/cis361.

[2] Ward JW. The hidden epidemic of hepatitis $C$ virus infection in the United States: occult transmission and burden of disease. Top Antivir Med 2013; 21:15-19.

[3] Maasoumy B, Wedemeyer $\mathrm{H}$. Natural history of acute and chronic hepatitis C. Best Pract Res Clin Gastroenterol 2012;26:401-412. doi: 10.1016/j.bpg. 2012.09.009.

[4] Mohd Hanafiah K, Groeger J, Flaxman AD, Wiersma ST. Global epidemiology of hepatitis $C$ virus infection: new estimates of age-specific antibody to $\mathrm{HCV}$ seroprevalence. Hepatology 2013;57:1333-1342. doi: 10.1002/hep.26141.

[5] Kanwal F, Hoang T, Kramer JR, Asch SM, Goetz MB, Zeringue Al. Increasing prevalence of $\mathrm{HCC}$ and cirrhosis in patients with chronic hepatitis $\mathrm{C}$ virus infection. Gastroenterology 2011;140:1182-1188. doi: 10.1053/j.gastro. 2010.12.032.

[6] Liang TJ, Ghany MG. Current and future therapies for hepatitis C virus infection. N Engl J Med 2013;368:1907-1917. doi: 10.1056/NEJMra1213651.

[7] Pawlotsky JM. New hepatitis C virus (HCV) drugs and the hope for a cure: concepts in anti-HCV drug development. Semin Liver Dis 2014;34:22-29. doi: $10.1055 / \mathrm{s}-0034-1371007$.

[8] Doyle JS, Aspinall E, Liew D, Thompson AJ, Hellard ME. Current and emerging antiviral treatments for hepatitis C infection. Br J Clin Pharmacol 2013;75: 931-943. doi: 10.1111/j.1365-2125.

[9] Dabbouseh N, Jensen D. Future therapies for chronic hepatitis C. Nat Rev Gastroenterol Hepatol 2013;10:268-276. doi: 10.1038/nrgastro.

[10] Afdhal N, Zeuzem S, Kwo P, Chojkier M, Gitlin N, Puoti MJJ, et al. Ledipasvir and sofosbuvir for untreated HCV genotype 1 infection. N Engl J Med 2014; 370:1889-1898. doi: 10.1056/NEJMoa1402454.

[11] Kanda T, Imazeki F, Yokosuka O. New antiviral therapies for chronic hepatitis C. Hepatol Int 2010;4:548-561. doi: 10.1007/s12072-010-9193-3.

[12] Thompson JR. Emerging therapeutic options for the management of hepatitis C infection. World J Gastroenterol 2014;20:7079-7088. doi: 10.3748/wjg. v20.i23.7079.

[13] Jeffers LJ. Treating hepatitis $C$ in African Americans. Liver Int 2007;27: 313-322. doi: 10.1111/j.1478-3231.2006.01438.x.

[14] Karoney MJ, Siika AM. Hepatitis C virus (HCV) infection in Africa: a review. Pan Afr Med J 2013;14:44. doi: 10.11604/pamj.

[15] Kinzie JL, Naylor PH, Nathani MG, Peleman RR, Ehrinpreis MN, Lybik M, et al. African Americans with genotype 1 treated with interferon for chronic hepatitis $C$ have a lower end of treatment response than Caucasians. J Viral Hepat 2001;8:264-269. doi: 10.1046/j.1365-2893.2001.00292.x.

[16] Jin R, Cai L, Tan M, Mchutchison JG, Thomas C, Howell CD. Optimum Ribavirin Exposure Overcomes Racial Disparity in Efficacy of Peginterferon and Ribavirin Treatment for Hepatitis C Genotype 1. Am J Gastroenterol 2012;107: 1675-1683. doi: 10.1038/ajg.2012.306.

[17] Flamm SL, Muir AJ, Fried MW, Reddy KR, Nelson DR, Bzowej NH, et al. Sustained Virologic Response Rates With Telaprevir-Based Therapy in Treatment-Naive Patients Evaluated by Race or Ethnicity. J Clin Gastroenterol 2015;49:336-344. doi: 10.1097/MCG.0000000000000150.

[18] Walzer N, Flamm SL. Pegylated IFN- $\alpha$ and ribavirin: emerging data in the treatment of special populations. Expert Rev Clin Pharmacol 2009;2: 67-76. doi: 10.1586/17512433.2.1.67.

[19] Crosse K, Umeadi OG, Anania FA, Laurin J, Papadimitriou J, Drachenberg C, et al. Racial differences in liver inflammation and fibrosis related to chronic hepatitis C. Clin Gastroenterol Hepatol 2004;2:463-468. doi: 10.1016/ S1542-3565(04)00162-4.
[20] Kallwitz ER, Layden-Almer ], Dhamija M, Berkes J, Guzman G, Lepe R, et al. Ethnicity and body mass index are associated with hepatitis $C$ presentation and progression. Clin Gastroenterol Hepatol 2010;8:72-78. doi: 10.1016/ j.cgh.

[21] Baran B, Gulluoglu M, Soyer OM, Ormeci AC, Gokturk S, Evirgen S, et al. Treatment failure may lead to accelerated fibrosis progression in patients with chronic hepatitis C. J Viral Hepat 2014;21:111-120. doi: 10.1111/jvh. 12127.

[22] Dieperink E, Pocha C, Thuras P, Knott A, Colton S, Ho SB. All-Cause Mortality and Liver-Related Outcomes Following Successful Antiviral Treatment for Chronic Hepatitis C. Dig Dis Sci 2014;59:872-880. doi: 10.1007/s10620014-3050-5.

[23] Morgan RL, Baack B, Smith BD, Yartel A, Pitasi M, Falck-ytter Y. Eradication of Hepatitis C Virus Infection and the Development of Hepatocellular Carcinoma. Ann Intern Med 2014;158:329-337. doi: 10.7326/0003-4819-1585-201303050-00005.

[24] Ogawa E, Furusyo N, Kajiwara E, Takahashi K, Nomura H, Maruyama T, et al. Efficacy of pegylated interferon alpha- $2 \mathrm{~b}$ and ribavirin treatment on the risk of hepatocellular carcinoma in patients with chronic hepatitis C: a prospective, multicenter study. J Hepatol 2013;58:495-501. doi: 10.1016/j.jhep.

[25] Poynard T, McHutchison J, Manns M, Trepo C, Lindsay K, Goodman Z, et al. Impact of pegylated interferon alfa- $2 b$ and ribavirin on liver fibrosis in patients with chronic hepatitis C. Gastroenterol 2002;122:1303-1313. doi: 10.1053 /gast.2002.33023.

[26] Veldt BJ, Heathcote EJ, Wedemeyer H, Reichen J, Hofmann WP, Zeuzem S, et al. Sustained Virologic Response and Clinical Outcomes in Patients with Chronic Hepatitis C and Advanced Fibrosis. Ann Intern Med 2007;147: 677-684. doi: 10.7326/0003-4819-147-10-200711200-00003.

[27] Singal AG, Pillai A, Tiro J. Early detection, curative treatment, and survival rates for hepatocellular carcinoma surveillance in patients with cirrhosis: a meta-analysis. PLoS Med 2014;11:e1001624. doi: 10.1371/journal.pmed. 1001624.

[28] Cozen ML, Ryan JC, Shen H, Lerrigo R, Yee RM, Sheen E, et al. Nonresponse to Interferon-alpha Based Treatment for Chronic Hepatitis C Infection Is Associated with Increased Hazard of Cirrhosis. PLoS One 2013;8:e61568. doi: 10.1371/journal.pone.0061568.

[29] McCombs J, Matsuda T, Tonnu-Mihara I, Saab S, Hines P, L'italien G, et al. The risk of long-term morbidity and mortality in patients with chronic hepatitis $\mathrm{C}$ : results from an analysis of data from a Department of Veterans Affairs Clinical Registry. JAMA Intern Med 2014;174:204-212. doi: 10.1001/ jamainternmed.

[30] Siddiqui F, Ehrinpreis MN, Janisse J, Dhar R, May E, Mutchnick MG. Demographics of a large cohort of urban chronic hepatitis $C$ patients. Hepatol Int 2008;2:376-381. doi: 10.1007/s12072-008-9086-x.

[31] Castera L. Non-invasive assessment of liver fibrosis in chronic hepatitis C. Hepatol Int 2011;5:625-634. doi: 10.1007/s12072-010-9240-0.

[32] Manning DS, Afdhal NH. Diagnosis and quantitation of fibrosis. Gastroenterology 2008;134:1670-1681. doi: 10.1053/j.gastro.

[33] Lin S, Xin ZH, Dong YN, Wang QJ, Jiang XJ, Zhan SH, et al. Performance of the aspartate aminotransferase-to-platelet ratio index for the staging of hepatitis C-related fibrosis: An updated meta-analysis. Hepatology 2011;53:726-736. doi: 10.1002/hep.24105.

[34] Sterling RK, Lissen E, Clumeck N, Sola R, Correa MC, Montaner J, et al. Development of a simple noninvasive index to predict significant fibrosis in patients with HIV/HCV coinfection. Hepatology 2006;43:1317-1325. doi: 10.1002/hep.21178.

[35] Pinzone MR, Zanghì AM, Rapisarda L, D'Agata V, Benanti F, Spartà D, et al. Cirrhotic patients are still at risk of developing hepatocellular carcinoma despite Interferon-induced sustained virological response. Eur Rev Med Pharmacol Sci 2014;18:11-15.

[36] Aleman S, Rahbin N, Weiland O, Davidsdottir L, Hedenstierna M, Rose N, et al. A risk for hepatocellular carcinoma persists long-term after sustained virologic response in patients with hepatitis C-associated liver cirrhosis. Clin Infect Dis 2013;57:230-236. doi: 10.1093/cid/cit234.

[37] Sato A, Sata M, Ikeda K, Kumada T, Izumi N, Asahina Y, et al. Clinical characteristics of patients who developed hepatocellular carcinoma after hepatitis $C$ virus eradication with interferon therapy: current status in Japan. Intern Med 2013;52:2701-2706. doi: 10.2169/internalmedicine.52.1180. 